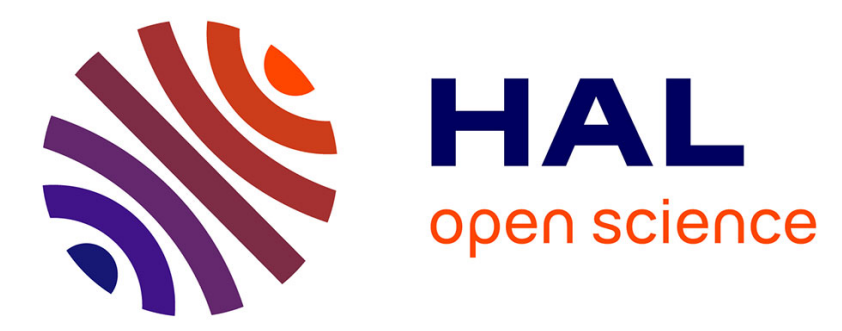

\title{
Integrated vehicle control through the coordination of longitudinal/lateral and vertical dynamics controllers: Flatness and LPV $/ \mathrm{H} \infty$ based design
}

Soheib Fergani, Lghani Menhour, Olivier Sename, Luc Dugard, Brigitte d'Andréa-Novel

\section{To cite this version:}

Soheib Fergani, Lghani Menhour, Olivier Sename, Luc Dugard, Brigitte d'Andréa-Novel. Integrated vehicle control through the coordination of longitudinal/lateral and vertical dynamics controllers: Flatness and LPV $/ \mathrm{H} \infty$ based design. International Journal of Robust and Nonlinear Control, 2017, 27 (18), pp.4992 -5007. 10.1002/rnc.3846 . hal-01509787

\section{HAL Id: hal-01509787 https://hal.science/hal-01509787}

Submitted on 24 Apr 2017

HAL is a multi-disciplinary open access archive for the deposit and dissemination of scientific research documents, whether they are published or not. The documents may come from teaching and research institutions in France or abroad, or from public or private research centers.
L'archive ouverte pluridisciplinaire HAL, est destinée au dépôt et à la diffusion de documents scientifiques de niveau recherche, publiés ou non, émanant des établissements d'enseignement et de recherche français ou étrangers, des laboratoires publics ou privés. 


\title{
Integrated vehicle control through the coordination of longitudinal/lateral and vertical dynamics controllers: Flatness and $\mathbf{L P V} / H_{\infty}$ based design
}

\author{
S. Fergani ${ }^{1 *}$, L. Menhour ${ }^{2}$, O. Sename ${ }^{1}$, L. Dugard ${ }^{1}$ and B. D’Andréa-Novel ${ }^{2}$ \\ ${ }^{1}$ Institut Supérieur de l'Aéronautique et de l'Espace (ISAE), Toulouse, 31055 France \\ ${ }^{2}$ Mines-ParisTech, CAOR-Centre de Robotique, Mathématiques et systèmes, France.
}

\begin{abstract}
SUMMARY
This paper deals with Global Chassis Control (GCC) of ground vehicles. It focuses on the coordination of suspensions and steering/braking vehicle controllers based on the interaction between the vertical and lateral behaviors of the vehicle. Indeed, the roll motion of the car can generate increasing load transfers that affect considerably the suspension system and vehicle stability. The load transfers can be described using the lateral acceleration. Then, the coordination is highlighted, in this work, through the relationship between the suspension behavior and the lateral acceleration in the framework of the Linear Paramter Varying (LPV) approach.

The proposed control law is designed in hierarchical way to improve the overall dynamics of the vehicle. This global control strategy includes two types controllers.

The first one is the longitudinal/lateral nonlinear Flatness controller. Based on the adequate choice of the flat outputs, the flatness proof of a 3DoF two wheels nonlinear vehicle model has been established. Then, the combined longitudinal and lateral vehicle control is designed. The algebraic estimation techniques have been used in order to have an accuracy estimation of the derivatives and filtering of the reference flat outputs. Such control strategy is developed in order to cope with coupled driving maneuvers like obstacle avoidance via steering control and stop-and-go control via braking or driving wheel torque.

The second part of the proposed strategy consists of the $L P V / \mathcal{H}_{\infty}$ suspension controller. This controller uses the lateral acceleration as a varying parameter to take into account the load transfers that affects directly the suspension system and therefore to achieve the desired performance.

The coordination between the vehicle vertical and lateral dynamics is highlighted in this study, and the $L P V / \mathcal{H}_{\infty}$ framework ensures a specific collaborative coordination between the suspension and the steering/braking controllers.

Simulations on a complex full vehicle model have been validated using experimental data obtained on-board vehicle, with an identification procedure on a real Renault Mégane Coupé.

Copyright (c) 2016 John Wiley \& Sons, Ltd.
\end{abstract}

Received ...

KEY WORDS: Global chassis Control, Braking, Steering, Suspension, LPV, Non linear flatness control, monitoring, $\mathcal{H}_{\infty}$, LMI. 
Table I. Variables of Vehicle.

\begin{tabular}{c|c|c}
\hline Symbol & Variable name & Unit \\
\hline$V_{x}$ & longitudinal speed & {$[\mathrm{m} / \mathrm{s}]$} \\
$V_{y}$ & lateral speed & {$[\mathrm{m} / \mathrm{s}]$} \\
$a_{x}$ & longitudinal acceleration & {$\left[\mathrm{m} / \mathrm{s}^{2}\right]$} \\
$a_{y}$ & lateral acceleration & {$\left[\mathrm{m} / \mathrm{s}^{2}\right]$} \\
$g$ & gravitational constant & {$\left[\mathrm{m} / \mathrm{s}^{2}\right]$} \\
$\mu$ & friction coefficient & \\
$\dot{\psi}$ & yaw rate & {$[\mathrm{rad} / \mathrm{s}]$} \\
$\psi$ & yaw angle & {$[\mathrm{rad}]$} \\
$\dot{\phi}_{v}$ & roll rate & {$[\mathrm{rad} / \mathrm{s}]$} \\
$\phi_{v}$ & vehicle roll angle & {$[\mathrm{rad}]$} \\
$\delta$ & steering angles & {$[\mathrm{rad}]$} \\
$T_{\omega}$ & braking/traction wheel torque & {$[\mathrm{Nm}]$} \\
$T_{m}$ & engine torque & {$[\mathrm{Nm}]$} \\
$T_{b}$ & braking torque & {$[\mathrm{Nm}]$} \\
$F_{y f}, F_{y r}$ & front and rear lateral tire forces & {$[\mathrm{N}]$} \\
$F_{s z_{i j}}$ & Suspension verticale forces & {$[\mathrm{N}]$} \\
$\alpha_{f}, \alpha_{r}$ & front and rear tire slip angles & {$[\mathrm{rad}]$} \\
$I_{z}$ & yaw moment of inertia & {$\left[\mathrm{Kg} \cdot \mathrm{m}^{-2}\right]$} \\
$I_{x}$ & moment of inertia about $x$ axis & {$\left[\mathrm{kg} \cdot \mathrm{m}^{2}\right]$} \\
$I_{x z}$ & moment of inertia about $x$ and $z$ axes & {$\left[\mathrm{kg} . \mathrm{m}^{2}\right]$} \\
$m_{2} m_{s}$ & mass and sprung mass of vehicle & {$[\mathrm{Kg}]$} \\
$C_{f}$ & front cornering stiffnesses & {$[\mathrm{N} / \mathrm{rad}]$} \\
$C_{r}$ & rear cornering stiffnesses & {$[\mathrm{N} / \mathrm{rad}]$} \\
$L_{f}$ & CoG to the front axles distances & {$[\mathrm{m}]$} \\
$L_{r}$ & CoG to the rear axles distances & {$[\mathrm{m}]$} \\
$R_{\omega}$ & tire radius & {$[\mathrm{m}]$} \\
$\phi_{r}$ & road bank angle & {$[\mathrm{rad}]$} \\
$T$ & sampling time & {$[\mathrm{s}]$} \\
& &
\end{tabular}

\section{NOTATIONS}

\section{INTRODUCTION}

The global chassis control of ground vehicles has been an important issue for the car's road safety in the last few years. In the last decade, lots of studies have treated this issue and proposed different solutions. Both passive safety systems (airbag, safety belt,...) and active safety systems (ABS, ESP,...) have been widely used by the automotive industry. The use of several actuators on the vehicle have allowed to control the different dynamics of the car, namely, the vertical, the lateral and the longitudinal ones. Many research works have tried to propose adequate solutions to the vehicle dynamics improvement issues, often through the global chassis control involving several actuators. In [1], a new design method of actuator intervention for trajectory tracking is proposed. In [2], an interesting nonlinear control law using suspension and braking actuators for commercial cars has been developed. Based on the LPV approaches, an integration of steering and braking controllers is proposed in [3], and comments on this integration are given in [4]. More recently, in [5], [6], [7], an LPV control structure that allows to coordinate several actuators and to improve different vehicle dynamics has been proposed. However, the suspension control coupled with longitudinal/lateral controller remains a challenging problem. Indeed, these problems are usually addressed separately. The proposed strategy is a hierarchical control structure that will improve the overall dynamics of the vehicle. It includes two controllers: the first one is the longitudinal/lateral nonlinear Flatness controller. Indeed, many works have proved that the non linear control strategies are efficient to

\footnotetext{
*Correspondence to: Département Conception et commande de véhicules AÃ@ Cronautiques et Spaciaux (DCAS) Institut Supérieur de l'Aéronautique et de l'Espace (ISAE), Toulouse, 31055 France. E-mail: soheib.fergani@ @isae.fr 
improve the lateral/longtudinal dynamics of the car. For this sake, the proposed control approach takes advantage of the flatness property (see [8], [9], [10]) to achieve a global linearization of the nonlinear system and of the algebraic estimation techniques for numerical differentiation and filtering of noisy signals [11], [12], [13]. Moreover, based on the adequate choice of the flat outputs, the flatness proof of a $3 \mathrm{DoF}$ two wheels nonlinear vehicle model is established. Thereafter, the combined longitudinal and lateral vehicle control is designed. The algebraic estimation techniques are used in order to have an accuracy estimation of the derivatives and filtering of the reference flat outputs. Such control strategy is developed in order to cope with coupled driving maneuvers like obstacle avoidance via steering control and stop-and-go control via braking or driving wheel torque. The second part of the proposed strategy consists in the full car $L P V / \mathcal{H}_{\infty}$ suspension controller. This controller uses the lateral acceleration as a varying parameter to take into account the load transfers that affects directly the suspension systems and therefore to achieve the desired performance. Indeed, the lateral motion of the vehicle is highly correlated to the vertical one through the load transfers induced by the roll motions. Based on this correlation, the $L P V / \mathcal{H}_{\infty}$ framework ensures the closed-loop stability for all parameter variations and gets the coordination between the vertical and the lateral dynamics performance objectives.

This paper is organized as follows: Section 3 presents the problem formation of the proposed integration. The design method of flat longitudinal/lateral control is addressed in Section 4. Section 5 gives the LPV/ $H_{\infty}$ suspension controller and the coordination strategy with flat longitudinal/lateral controller. In Section 6, the performance of the proposed integration control strategy are shown through simulation results. Finally, conclusions and future work are stated in Section 7.

\section{PROBLEM STATEMENT OF THE INTEGRATION OF THE NON LINEAR AND THE LPV CONTROLLERS}

The diagram in Fig. 1 describes the integration of the two proposed advanced controllers. In fact, the lateral acceleration controlled with the non linear flatness controller is used to deduce the varying parameters that schedules the $\mathrm{LPV} / H_{\infty}$ suspension controller for the vertical dynamics.

The objective of this integrated controller is to improve the vehicle handling and safety. More precisely, the control of longitudinal and lateral motions has a key role to handle some critical coupled driving maneuvers such as the obstacle avoidance via steering control. Simultaneously, the passengers comfort and car roadholding will enhance the vertical dynamics considering the inherent intercorrelation with the lateral ones. Therefore, the coordination in between the 3 main dynamics of the vehicle is established to achieve the desired performance objectives.

It is worth noting that since we are mainly interested by the load transfers caused by the roll dynamics generated, the following relationship between the vertical and lateral dynamics of the vehicle is considered:

$$
\theta=\frac{z_{d e f_{f l}}-z_{d e f_{f r}}+z_{d e f_{r l}}-z_{d e f_{r r}}}{t_{f}}-\frac{m_{s} a_{y} h}{k_{t}}
$$

where $\theta$ : is the roll motion of the vehicle, $a_{y}$ is the lateral acceleration (more details on the relationship between the vertical and the lateral dynamics are given in the following sections), $z_{\text {def }_{i j}}$ : is the suspension deflections ( $i$ : front or rear, $j$ : left or right), $k_{t}:$ is the tire stiffness.

On the other hand, the lateral and the vertical dynamics of the vehicle are correlated through the lateral and vertical tire forces as, (see [14] for more details):

$$
F_{i y}\left(\beta_{i}\right)=\operatorname{Sign}\left(\beta_{i}\right) F_{i z} \mu\left(\beta_{i}\right)
$$

where $i$ : is the index for front and rear wheels, $F_{i y}$ : is the lateral force, $\beta_{i}:$ is the sideslip, $F_{i z}$ : is the vertical force and $\mu\left(\beta_{i}\right)$ : is the road friction. Furthermore, this relationship can be observed also in the load transfer depending on the roll dynamic and the lateral acceleration $a_{y}$ (notice that 


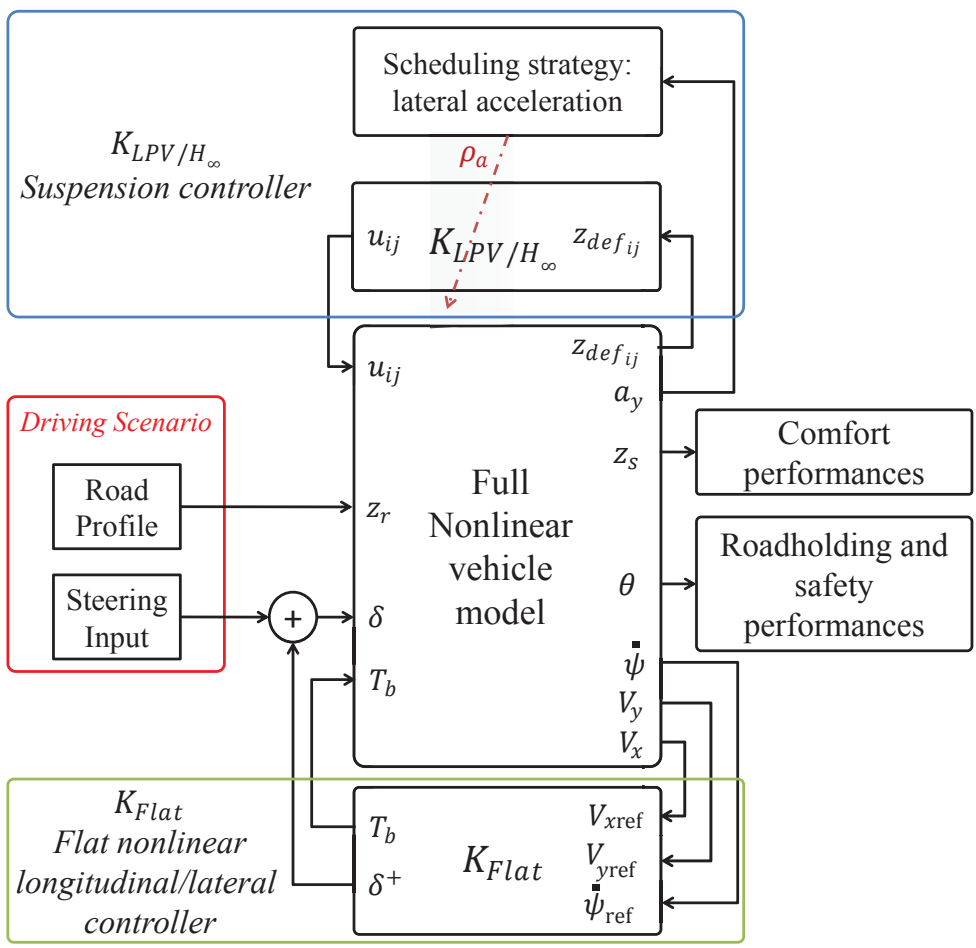

Figure 1. Diagram block of the integration strategy

$F y=m_{s} a_{y} K$, where $K$ : is a constant coefficient) as follows,(see [15]):

$$
\left\{\begin{aligned}
\Delta F z & =\left(F_{z_{f l}}+F_{z_{r l}}-F_{z_{f r}}-F_{z_{r r}}\right) \\
& =\left(m_{s_{f l}}+m_{s_{r l}}-m_{f r}-m_{s_{r r}}\right) 2 S_{1} g \theta+2 S_{2} a_{y} m_{s} / l
\end{aligned}\right.
$$

where $S_{1}=\frac{k_{f}}{t_{f}}+\frac{k_{r}}{t_{r}}, S_{2}=\frac{l_{f} h}{t_{f}}+\frac{l_{r} h}{t_{r}}$. It is clear that the load transfer generated by the vehicle bounce are largely influenced by the dynamics of the lateral acceleration, since, as emphasised in (1) the roll motion is also directly linked to $a_{y}$.

Based on the lateral acceleration, the following scheduling parameter is proposed as:

$$
\rho_{a}=\left|\frac{a_{y}}{a_{y_{\max }}}\right|
$$

The proposed strategy aims at enhancing the vehicle dynamics by applying, on the full non linear model of the vehicle, two kind of controllers acting simultaneously on the vertical dynamics (the Linear Parameters Varying control) and on the lateral/longitudinal dynamics (the non linear Flatness based control). An integration of these two control laws is achieved trough the existing correlations between the vehicle vertical, lateral and longitudinal dynamics.

\section{FLATNESS-BASED NONLINEAR CONTROL}

In this section, the design of the non linear longitudinal/lateral flatness control is described. The controller design structure is summarized in Fig. 2. This scheme summarizes the flatness control design structure. The non linear Flatness controller $K_{\text {Flat }}$ uses the longitudinal velocity $v_{x}$, the lateral velocity $v_{y}$ and the yaw rate $\dot{\psi}$ as inputs. It provides the braking torques $T_{b}$ and the corrective steering angle $\delta^{+}$that stabilize the vehicle in several driving situations. 


$$
\dot{x}=f(x, t)+g(x) u+g_{1} u_{1} u_{2}+g_{2} u_{2}^{2}
$$

where $x=\left[\begin{array}{c}V_{x} \\ V_{y} \\ \dot{\psi}\end{array}\right], u=\left[\begin{array}{c}u_{1} \\ u_{2}\end{array}\right], f(x, t)=\left[\begin{array}{c}\dot{\psi} V_{y}-\frac{I_{r}}{m_{s} R}\left(\dot{\omega}_{r}+\dot{\omega}_{f}\right) \\ -\dot{\psi} V_{x}+\frac{1}{m_{s}}\left(-C_{f}\left(\frac{V_{y}+L_{f} \dot{\psi}}{V_{x}}\right)-C_{r}\left(\frac{V_{y}-L_{r} \dot{\psi}}{V_{x}}\right)\right) \\ \frac{1}{I_{z}}\left(-L_{f} C_{f}\left(\frac{V_{y}+L_{f} \dot{\psi}}{V_{x}}\right)+L_{r} C_{r}\left(\frac{V_{y}-L_{r} \dot{\psi}}{V_{x}}\right)\right)\end{array}\right]$ and $g(x, t)=\left[\begin{array}{cc}\frac{1}{m_{s} R} & \frac{C_{f}}{m_{s}}\left(\frac{V_{y}+L_{f} \dot{\psi}}{V_{x}}\right) \\ 0 & \left(C_{f} R-I_{r} \dot{\omega}_{f}\right) / m_{s} R \\ 0 & \left(L_{f} C_{f} R-L_{f} I_{r} \dot{\omega}_{f}\right) / I_{z} R\end{array}\right]$.

where the longitudinal motion is controlled via the wheels torques $u_{1}=T_{i_{\omega}}=T_{i_{m}}-T_{i_{b}}$, where $T_{i_{m}}=0$ (no traction is considered here) and $T_{i_{b}}$ the braking torque for each wheel, and the lateral motion is controlled via the corrective steering angle $u_{2}=\delta^{+}$. The second order terms $u_{1} u_{2}$ and $u_{2}^{2}$ are neglected because of their small magnitude. More details on the previously used model

\footnotetext{
${ }^{\dagger}$ See [16] for details on these models.
} 
can be found in [16], [17].

For more details see 8.

\subsection{Synthesis of the Flatness-based longitudinal/lateral control}

Despite these simplifications some coupled behaviors are kept as shown by the functions $f(x, t)$ and $g(x, t)$. Eq. (6) becomes

$$
\dot{x}=f(x, t)+g(x, t) u
$$

Let us remind that the differential flatness approach of nonlinear systems in a differential algebraic context was introduced in [8], [9], [10]. Indeed, the necessary information to run the dynamic behavior of a real system are easily expressed by the appropriate flat outputs. Numerous engineering real applications using flat systems are already handled in the literature (see [9], [10], [18], [19]). Such an approach is also used to manage the coupled nonlinear vehicle control [20], [17] and underwater vehicles [21] (More application examples of the flatness approach can be found in [22], [23]).

Here, since it is well adapted to tracking control problems, the flatness approach is used to deal with a combined control of longitudinal and lateral vehicle motions using braking on each wheel. The following design problem and flatness property are used to establish the flatness of a 3DoF nonlinear vehicle model. Subsequently, the main objective of this section is presented.

\section{Theorem 1}

The following outputs:

$$
\left\{\begin{array}{l}
y_{1}=V_{x} \\
y_{2}=L_{f} m_{s} V_{y}-I_{z} \dot{\psi}
\end{array}\right.
$$

are flat outputs for the system (7) (details in [19]).

\section{Remark 1}

The first flat output $y_{1}$ is the longitudinal speed and the second one $y_{2}$ is the angular momentum of a point on the axis between the centers of the front and rear axles.

\section{Proof}

The flatness control is established thanks to the following flatness property [10], [22].

The objective is to show the flatness of model (7) with outputs (8) according to the flatness property 37. Then, after some algebraic manipulations we obtain:

$$
\left\{\begin{aligned}
x= & {\left[\begin{array}{c}
V_{x} \\
V_{y} \\
\dot{\psi}
\end{array}\right] } \\
= & A\left(y_{1}, y_{2}, \dot{y}_{2}\right) \\
= & {\left[\begin{array}{c}
\frac{y_{2}}{L_{1} m_{s}}-\left(\frac{I_{z}}{L_{1} m_{s}}\right)\left(\frac{y_{1} m_{s} y_{1} \dot{y}_{2}+C_{r}\left(L_{1}+L_{2}\right) y_{2}}{C_{r}\left(L_{1}+L_{2}\right)\left(I_{z}-L_{2} L_{1} m_{s}\right)+\left(L_{1} m_{s} y_{1}\right)^{2}}\right) \\
-\left(\frac{L_{1} m_{s} y_{1} \dot{y}_{2}+C_{r}\left(L_{1}+L_{2}\right) y_{2}}{C_{r}\left(L_{1}+L_{2}\right)\left(I_{z}-L_{2} L_{1} m_{s}\right)+\left(L_{1} m_{s} y_{1}\right)^{2}}\right)
\end{array}\right] }
\end{aligned}\right.
$$

and

$$
\left[\begin{array}{l}
\dot{y}_{1} \\
\ddot{y}_{2}
\end{array}\right]=\Delta\left(y_{1}, y_{2}, \dot{y}_{2}\right)\left(\begin{array}{l}
u_{1} \\
u_{2}
\end{array}\right)+\Phi\left(y_{1}, y_{2}, \dot{y}_{2}\right)
$$

where

$$
\Delta=\left[\begin{array}{ll}
\Delta_{11} & \Delta_{12} \\
\Delta_{21} & \Delta_{22}
\end{array}\right]
$$


and

$$
\Phi=\left[\begin{array}{l}
\Phi_{1} \\
\Phi_{2}
\end{array}\right]
$$

which is equivalent to

$$
\left[\begin{array}{l}
u_{1} \\
u_{2}
\end{array}\right]=\Delta^{-1}\left(y_{1}, y_{2}, \dot{y}_{2}\right)\left(\left[\begin{array}{l}
\dot{y}_{1} \\
\ddot{y}_{2}
\end{array}\right]-\Phi\left(y_{1}, y_{2}, \dot{y}_{2}\right)\right)
$$

For details concerning $\Delta$ and $\Phi$, see [17]. Thus, the control inputs of the considered systems can be written as follows:

$$
\left\{\begin{aligned}
u & =\left[\begin{array}{c}
T_{\omega} \\
\delta
\end{array}\right] \\
& =B\left(y_{1}, \dot{y}_{1}, y_{2}, \dot{y}_{2}, \ddot{y}_{2}\right) \\
& =\Delta^{-1}\left(y_{1}, y_{2}, \dot{y}_{2}\right)\left(\left[\begin{array}{c}
\dot{y}_{1} \\
\ddot{y}_{2}
\end{array}\right]-\Phi\left(y_{1}, y_{2}, \dot{y}_{2}\right)\right)
\end{aligned}\right.
$$

with $r_{x}=1$ and $r_{u}=2$. Finally, the system (6) is flat system with outputs (8), then, the outputs (8) are called flat outputs.

Then, in order to track the desired outputs $y_{1}^{r e f}$ and $y_{2}^{r e f}$, the outputs are rewritten as follows:

$$
\left[\begin{array}{c}
\dot{y}_{1} \\
\ddot{y}_{2}
\end{array}\right]=\left[\begin{array}{c}
\dot{y}_{1}^{r e f}+K_{1}^{1} e_{y_{1}}+K_{1}^{2} \int e_{y_{1}} d t \\
\ddot{y}_{2}^{r e f}+K_{2}^{1} e_{y_{2}}+K_{2}^{2} \int e_{y_{2}} d t+K_{2}^{3} \dot{e}_{y_{2}}
\end{array}\right]
$$

where, $e_{y_{1}}=y_{1}^{r e f}-y_{1}=V_{x}^{r e f}-V_{x}$ and $e_{y_{2}}=y_{2}^{r e f}-y_{2}$. The choice of the gain parameters $K_{1}^{1}$, $K_{1}^{2}, K_{2}^{1}, K_{2}^{2}$ and $K_{2}^{3}$ is then straightforward.

\subsection{Algebraic nonlinear estimation}

It should be pointed out that the control law (14) contains derivatives of reference signals which are computed from measurements such as $V_{x}^{r e f}, V_{y}^{r e f}, \dot{\psi}^{\text {ref }}$ and the derivatives of the measured front and rear rotational speed wheels $\omega_{f}$ and $\omega_{r}$. In order to minimize the effect of the noise on these derivatives, the numerical differentiation based on an algebraic nonlinear estimation $*$ is proposed. This estimation is performed using the recent advances in [11], [12], which yield efficient real-time filters ${ }^{\S}$. The following formulae (see, e.g., [26]) may be used:

- Denoising:

$$
\hat{y}(t)=\frac{2 !}{T^{2}} \int_{t-T}^{t}(2 T-3 \tau) y(\tau) d \tau
$$

- The numerical differentiation of a noisy signal:

$$
\hat{\dot{y}}(t)=-\frac{3 !}{T^{3}} \int_{t-T}^{t}(T-2 \tau) y(\tau) d \tau
$$

Note that the sliding time window $[t-T, t]$ may be quite short.

The equations (18) and (19) illustrate the derivatives and filtering of longitudinal speed, lateral speed, yaw rate and wheels rotation speeds. The results of this application are used as reference signals in the different parts of the control strategy in (20).

\footnotetext{
${ }^{\ddagger}$ See [19], [24], [25] for previous successful applications to intelligent transportation systems.

$\S$ The above estimation methods are not of asymptotic type and do not require any statistical knowledge of the corrupting noises (see [13] for details). 
- The estimated derivatives $\hat{\dot{V}}_{x}^{r e f}, \hat{\dot{V}}_{y}^{r e f}, \hat{\ddot{\psi}}^{r e f}, \hat{\dot{\omega}}_{f}$ and $\hat{\dot{\omega}}_{r}$ are performed as follows:

$$
\left[\begin{array}{c}
\hat{\dot{V}}_{x}^{\text {ref }} \\
\hat{\dot{V}}_{y}^{r e f} \\
\ddot{\dot{\psi}}^{\text {ref }} \\
\hat{\dot{\omega}}_{f} \\
\dot{\hat{\omega}}_{r}
\end{array}\right]=-\frac{3 !}{T^{3}} \int_{t-T}^{t}(2 T(t-\tau)-T)\left[\begin{array}{c}
V_{x}^{\text {ref }} \\
V_{y}^{\text {ref }} \\
\dot{\psi}^{\text {ref }} \\
\omega_{f} \\
\omega_{r}
\end{array}\right] d \tau
$$

- The filtering $\hat{\dot{V}}_{x}^{r e f}, \hat{\dot{V}}_{y}^{r e f}$ and $\hat{\dot{\psi}}^{r e f}$ are performed as follows:

$$
\left[\begin{array}{c}
\hat{V}_{x}^{r e f} \\
\hat{V}_{y}^{r e f} \\
\dot{\psi}^{r e f}
\end{array}\right]=\frac{2 !}{T^{2}} \int_{t-T}^{t}(3(t-\tau)-T) y(\tau)\left[\begin{array}{c}
V_{x}^{r e f} \\
V_{y}^{r e f} \\
\dot{\psi}^{\text {ref }}
\end{array}\right] d \tau
$$

Finally, the equation of the coupled nonlinear control obtained from equations (14), (15) is as follows:

$$
\begin{aligned}
u & =\left[\begin{array}{c}
T_{\omega} \\
\delta
\end{array}\right] \\
& =\Delta^{-1}\left(y_{1}, y_{2}, \dot{y}_{2}\right)\left(-\Phi\left(y_{1}, y_{2}, \dot{y}_{2}\right)+\left[\begin{array}{c}
\hat{y}_{1}^{r e f}+K_{1}^{1} \hat{e}_{y_{1}}+K_{1}^{2} \int \hat{e}_{y_{1}} d t \\
\hat{\ddot{y}}_{2}^{r e f}+K_{2}^{1} \hat{e}_{y_{2}}+K_{2}^{2} \int \hat{e}_{y_{2}} d t+K_{2}^{3} \hat{\dot{e}}_{y_{2}}
\end{array}\right]\right)
\end{aligned}
$$

where $\hat{e}_{y_{i}}$ : are the outputs errors estimations.

\section{5. $L P V / \mathcal{H}_{\infty}$ BASED SUSPENSION CONTROL}

The $L P V / \mathcal{H}_{\infty}$ suspension control shown on Fig. 3 is designed using a 7DoF vehicle model (21). It includes the following dynamics like: chassis acceleration $\ddot{z}_{s}$, four wheels accelerations $\ddot{z}_{u s_{i j}}$, roll bounce acceleration $\ddot{\theta}$, pitch acceleration $\ddot{\phi}$.

The equations describing the vertical 7DOF model are giving as follows:

$$
\left\{\begin{array}{l}
\ddot{z}_{s}=-\left(F_{s z_{f}}+F_{s z_{r}}+F_{d z}\right) / m_{s} \\
\ddot{z}_{u s_{i j}}=\left(F_{s z_{i j}}-F_{t z_{i j}}\right) / m_{u s_{i j}} \\
\ddot{\theta}=\left(\left(F_{s z_{r l}}-F_{s z_{r r}}\right) t_{r}+\left(F_{s z_{f l}}-F_{s z_{f r}}\right) t_{f}+m_{s} h \dot{V}_{y}\right) / I_{x} \\
\ddot{\phi}=\left(F_{s z_{f}} l_{f}-F_{s z_{r}} l_{r}-m_{s} h \dot{V}_{x}\right) / I_{y}
\end{array}\right.
$$

\section{1. $L P V / H_{\infty}$ suspension controller design}

The suspension control design aims at providing the performance adaptation thanks to the previously defined varying parameter $\rho_{a}$ in Section. 4 . It is presented in the framework of the $\mathcal{H}_{\infty}$ control approach including the parameter varying weighting functions and is summarized as follows:

Indeed, according to the control scheme proposed in Fig. 3, let us define:

- $W_{z_{s}}=\left(1-\rho_{a}\right) \frac{s^{2}+2 \xi_{11} \Omega_{11} s+\Omega_{11}^{2}}{s^{2}+2 \xi_{12} \Omega_{12} s+\Omega_{12}^{2}}$ is selected to reduce the bounce amplification of the suspended mass motion $\left(z_{s}\right)$ between $[0,12] H z$.

- $W_{\theta}=\left(\rho_{a}\right) \frac{s^{2}+2 \xi_{21} \Omega_{21} s+\Omega_{21}^{2}}{s^{2}+2 \xi_{22} \Omega_{22} s+\Omega_{22}^{2}}$ which attenuates the roll bounce amplification in low frequencies.

- $W_{u}=3.10^{-2}$ which limits the control signal.

Remark 2

It should be noticed that the parameters of these weighting functions are obtained using genetic algorithm optimization as in [27]. 


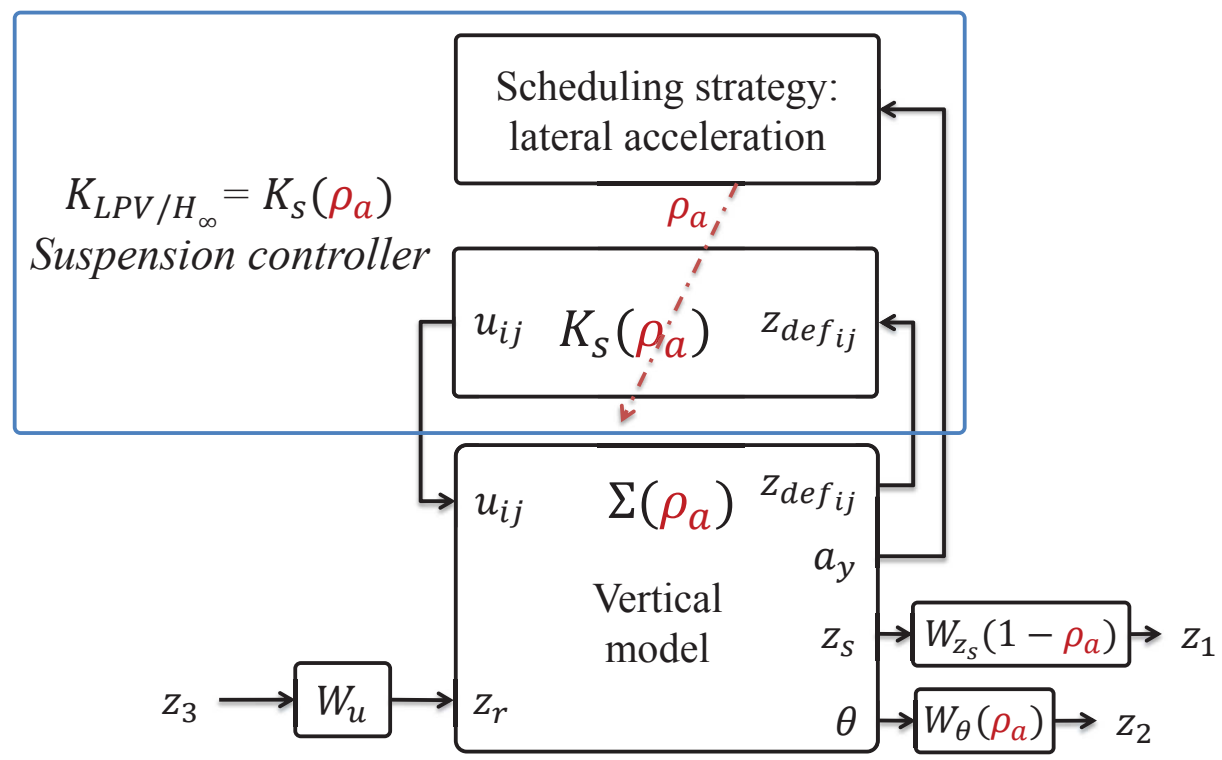

Figure 3. Diagram block of the $\mathrm{LPV} / H_{\infty}$ suspension control

The main objective of the proposed strategy is to improve the passengers comfort (when there is no risk) and the vehicle road holding. The use of the $H_{\infty}$ control allows to provide a robust solution that handles the most critical case (dangerous driving situations). Also, the LPV framework ensures a smart adaption of the objective performances to the different driving situations.

According to Fig. 3, the following parameter dependent generalized system $\left(\Sigma\left(\rho_{a}\right)\right)$ is obtained:

$$
\Sigma\left(\rho_{a}\right):\left\{\begin{array}{l}
\dot{\xi}=A\left(\rho_{a}\right) \xi+B_{1}\left(\rho_{a}\right) \tilde{w}+B_{2} u \\
\tilde{z}=C_{1}\left(\rho_{a}\right) \xi+D_{11} \tilde{w}+D_{12} u \\
y=C_{2} \xi+D_{21} \tilde{w}+D_{22} u
\end{array}\right.
$$

where $\xi=\left[\begin{array}{ll}\mathcal{X}_{\text {vert }} & \mathcal{X}_{w}\end{array}\right]^{T}, \tilde{z}=\left[\begin{array}{lll}z_{1} & z_{2} & z_{3}\end{array}\right]^{T}, \tilde{w}=\left[\begin{array}{lll}z_{r_{i j}} & F_{d x, y, z} & m_{d x, y}\end{array}\right]^{T}, y=z_{d e f_{i j}}, u=u_{i j}^{H \infty}$ and $\mathcal{X}_{w}$ are the vertical weighting functions states.

Such a choice allows the following scheduling policy:

- In normal driving situations, the lateral acceleration is low, consequently, the parameter $\rho_{a}$ tends to 0 . In this case, the $\mathrm{LPV} / H_{\infty}$ suspension control focuses on improving passengers comfort by reducing the chassis displacement and accelerations.

- when $\rho_{a} \longrightarrow 1$, the roll motion caused by high lateral accelerations is penalized to reduce the load transfer bounce and then enhance the roadholding, so the stability and safety of the vehicle are improved.

Now, the varying parameter $\rho_{a}$ is considered bounded: $\rho_{a} \in[0,1]$. Indeed, the LPV system (Eq. 22) includes a single scheduling parameter $\rho_{a} \in \mathcal{P}_{\rho}$, s.t.

$$
\rho_{a} \in\left[\begin{array}{ll}
\rho_{a} & \bar{\rho}_{a}
\end{array}\right] \text {, where, } \underline{\rho}_{a}=\rho_{\min }=0 \text { and } \bar{\rho}_{a}=\rho_{\max }=1
$$

and can be described as a polytopic system, i.e, a convex combination of the systems defined at each vertex of a polytope defined by the bounds of the varying parameter. The synthesis of the two sub-system controllers is performed in the framework of the $H_{\infty}$ control of polytopic suspensions) as follow:s

Let assume that $x \in X \in \mathbb{R}^{n}, z \in Z \in \mathbb{R}^{n_{z}}, y \in Y \in \mathbb{R}^{n_{y}}, \tilde{w} \in W \in \mathbb{R}^{n_{\tilde{w}}}$ and $u \in U \in \mathbb{R}^{n_{u}}$ and $\rho_{a} \in \mathcal{P}_{\rho}$. The considered LPV generalized plant that copes to the polytopic approach is:

$$
\left[\begin{array}{c}
\dot{\xi} \\
\hline z \\
y
\end{array}\right]=\left[\begin{array}{c|cc}
A\left(\rho_{a}\right) & B_{1}\left(\rho_{a}\right) & B_{2} \\
\hline C_{1}\left(\rho_{a}\right) & D_{11}\left(\rho_{a}\right) & D_{12} \\
C_{2} & D_{21} & D_{22}
\end{array}\right]\left[\begin{array}{c}
\xi \\
\hline \tilde{w} \\
u
\end{array}\right]
$$


According to this general plant formulation, the LPV controller $K_{s}\left(\rho_{a}\right)$ designed is defined as,

$$
\left[\begin{array}{c}
\dot{x_{c}} \\
u
\end{array}\right]=\left[\begin{array}{cc}
A_{c}\left(\rho_{a}\right) & B_{c}\left(\rho_{a}\right) \\
C_{c}\left(\rho_{a}\right) & D_{c}\left(\rho_{a}\right)
\end{array}\right]\left[\begin{array}{c}
x_{c} \\
y
\end{array}\right]
$$

where $x_{c} \in X_{c} \in \mathbb{R}^{n}$ are the controller states, $u \in U \in \mathbb{R}^{n_{u}}, y \in Y \in \mathbb{R}^{n_{y}}$.

According to the varying parameter $\rho_{a} \in \mathcal{P}_{\rho} \in \mathbb{R}^{l}$, the reconstruction of the LPV polytopic controller, composed by 2 vertices (see [27]), can be expressed as:

$$
K\left(\rho_{a}\right)=\sum_{i=1}^{2} \alpha_{i}(\alpha)\left[\begin{array}{ll}
A_{c_{i}} & B_{c_{i}} \\
C_{c_{i}} & D_{c_{i}}
\end{array}\right]
$$

where $c_{i}$ define the controller at each vertex of the parameter polytope and where,

$$
\begin{gathered}
\alpha_{i}\left(\rho_{a}\right):=\frac{\prod_{k=1}^{2}\left|\rho_{k}-\mathcal{C}\left(\omega_{i}\right)_{k}\right|}{\prod_{k=1}^{2}\left(\bar{\rho}_{k}-\underline{\rho}_{k}\right)}, i=1, \ldots, N \\
\alpha_{i}\left(\rho_{a}\right) \geq 0 \text { and } \sum_{i=1}^{2} \alpha_{i}\left(\rho_{a}\right)=1
\end{gathered}
$$

Remark 3

It is worth to stress that the use of only one varying parameter reduce the conservatism of the considered controller and the make it easy to implement the proposed.

\section{SIMULATION RESULTS OF THE INTEGRATED STRATEGY}

In this section, we present the simulation results obtained with integrated controller and considering a nonlinear vehicle model including vertical and suspension model (more details in [28].

\section{Remark 4}

It is worth reminding that the simulation model was validated by experimental tests and implementations on a real vehicle, the Renault Mégane Coupé (more details in [29]). All the control design model are driven (linearization) based on that validated model.

This following scenario is used to emphasize the efficiency of the integrated strategy to operate in critical driving situations. In the beginning of the scenario, the vehicle runs at $90 \mathrm{~km} / \mathrm{h}$ in straight line on a wet road $(\mu=0.5)$. The driver performs a line change maneuver between $t=0.5 \mathrm{~s}$ and $t=2 \mathrm{~s}$. First, a $5 \mathrm{~cm}$ bump occurs on the left wheels (from $t=0.5 \mathrm{~s}$ to $t=1 \mathrm{~s}$ ) then another one between $t=3 \mathrm{~s}$ and $t=4 \mathrm{~s}$. In addition, a lateral wind is generating an undesirable yaw moment (from $t=2 \mathrm{~s}$ to $t=2.5 \mathrm{~s}$ ).

Fig. 4 shows the performance results of the flat controller to track the desired flat outputs $y_{1}$ and $y_{2}$. Moreover, the steering angle and braking torque needed to track the flat outputs are presented in Fig. 5.

Figs. 6 and 7 show the lateral acceleration of the vehicle and the scheduling parameter used by the LPV/ $H_{\infty}$, respectively, while performing the proposed scenario. It can be seen that the lateral acceleration rises when performing the line change maneuver (lateral dynamics strongly excited), at the same time the considered varying parameter $\rho_{a}$ value increases to achieve properly the performance scheduling task.

The chassis displacement (representing passengers comfort) and roll bounce motion of the vehicle (representing the vehicle roadholding) are given in Figs. 8 and 9.

By calculating the RMS of the roll dynamics and the chassis displacement dynamics, one can

\footnotetext{
T The control law is obtained from the integrated of flat longitudinal/lateral control and LPV/ $H_{\infty}$ suspension control. 

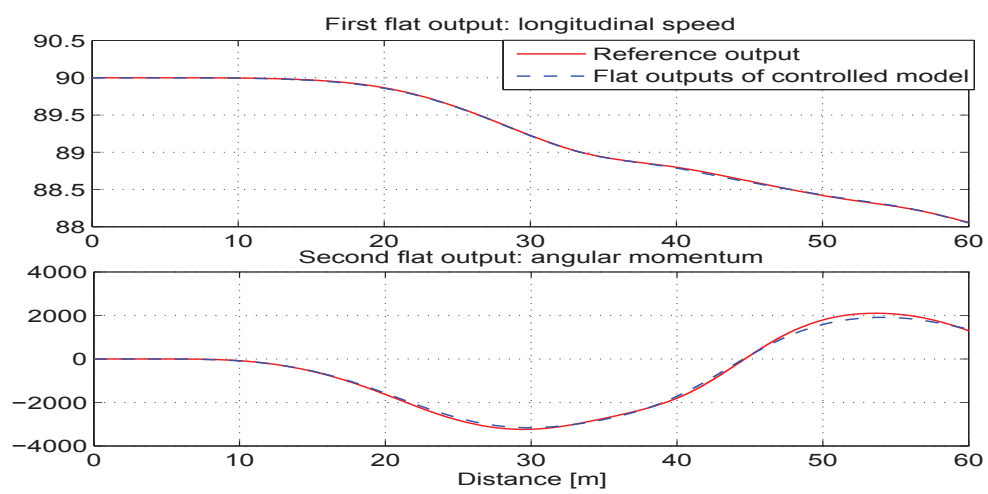

Figure 4. Flat outputs: reference and controlled model
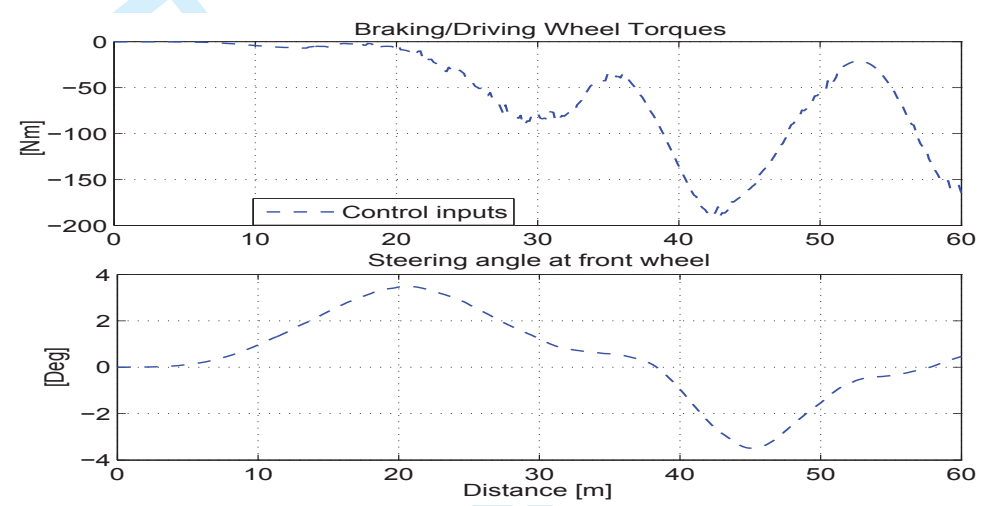

Figure 5. Coupled longitudinal/lateral flat control signals

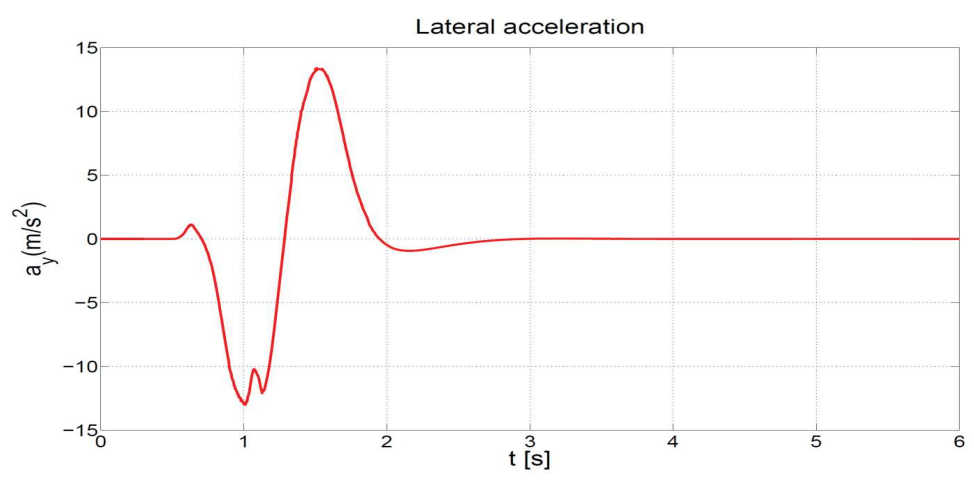

Figure 6. Lateral acceleration

clearly see the performance adaption to the driving situation brought by the proposed strategy. Indeed, when the driver perform the line change and face the first bump, the driving situation is dangerous and the lateral acceleration increases (as in Fig. 6). Therefore, $\rho_{a} \longrightarrow 1$ (as shown in Fig. 7) and the suspension control is adapted to penalize the roll dynamics (RMS (Real Mean Square value) of the roll is enhanced $48 \%$ ) and reduce the load transfer and improve the vehicle safety and handling (see Section 5) and relax the weighting on the chassis displacement (enhanced 28\%). Also, it can be clearly seen in Fig. 8 that after the line change and when the vehicle encounters the bump in a straight road, the riving situation is normal, $\rho_{a} \longrightarrow 0$ and the suspension control focuses on improving passengers comfort by reducing the chassis displacement $z_{s}$ (chassis displacement 


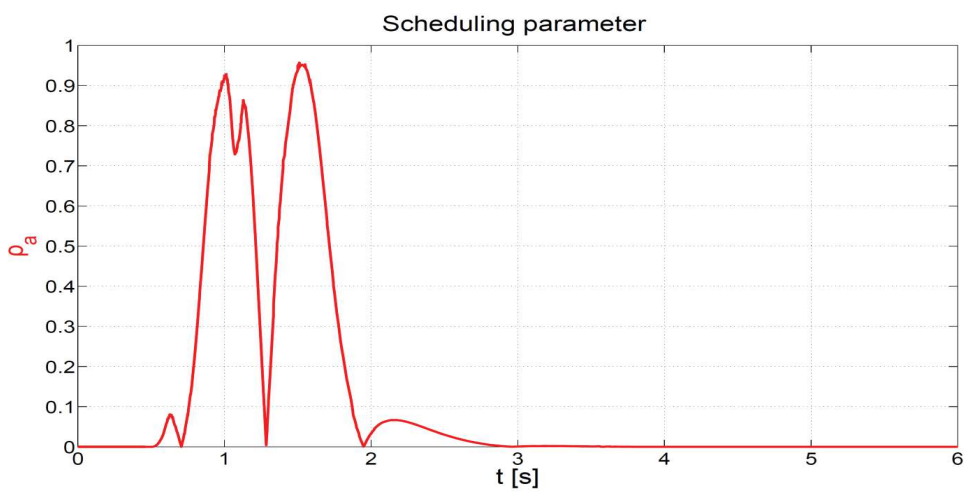

Figure 7. Scheduling parameter $\rho_{a}$

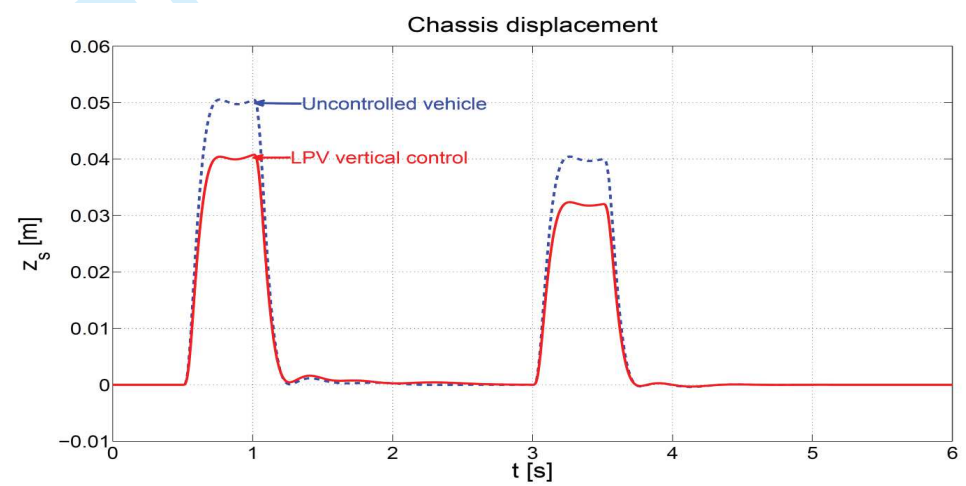

Figure 8. Chassis displacement $z_{s}$

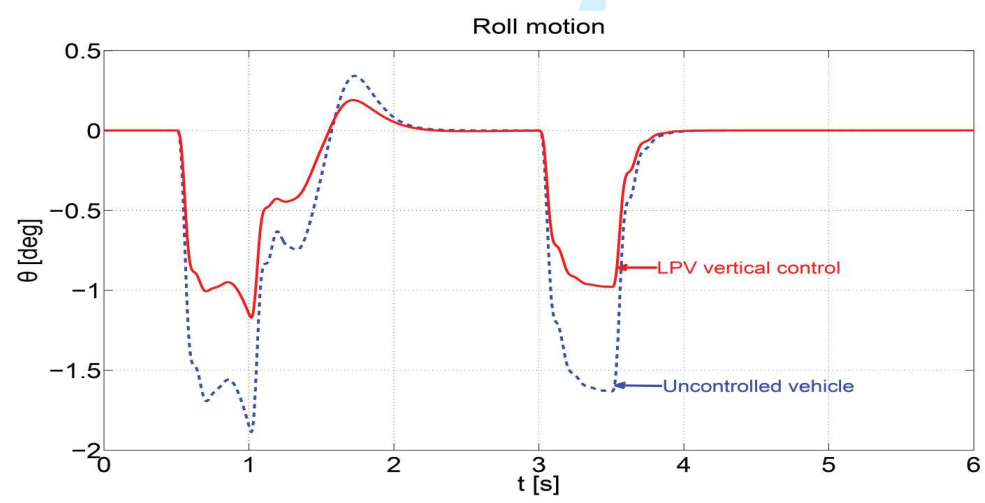

Figure 9. Roll angle $\theta$

enhanced by $41 \%$ ).

Other results on the stability of the controlled vehicle are highlighted in Fig. 10. In fact, the controlled vehicle model remains inside of the stability region (defined sideslip dynamics of the car) even in the critical driving situations, however, the uncontrolled model operates outside the stability region. These results confirm the ability of the proposed integration strategy to keep the controlled vehicle model more stable.

The obtained results confirm the efficiency of this control strategy to enhance the vehicle dynamics and overcome the dangerous driving situations. 


\section{CONCLUSION}

In this study, a novel integration strategy of two advanced vehicle controllers is developed. Firstly, the lateral/longitudinal flatness control and the $\mathrm{LPV} / H_{\infty}$ vertical dynamics control have been designed respectively. Then, an innovative coordination method between the two strategies has been proposed to establish the collaborative work of the two controllers. This integration aims to improve the handling and safety of the vehicle, and simultaneously ensures a control coordination of the several vehicle dynamics to perform combined maneuvers.

Simulation results asses the performance of this collaborative strategy for enhancing the longitudinal, lateral and vertical dynamics and have shown the efficiency of the proposed approach. Also, using the LPV coordination framework allows to simplify the implementation procedure. 


\section{APPENDIX. RECALL ON FLATNESS BASED ALGEBRAIC THEORY}

The idea of differentially flat systems appeared at the nineties of the last century. Flat systems are an important subclass of nonlinear control systems introduced via differential-algebraic methods, defined in a differential geometric framework.

The concept of differential algebra is used to define the flat systems, as introduced in [9] and later using Lie-Bäcklund transformations. Indeed, the system is said to be flat if one can find a set of variables, called the flat outputs, such that the system is (non-differentially) algebraic over the differential field generated by the set of flat outputs. It means that a system is flat if we can find a set of outputs, such that all states and inputs can be determined from these outputs without integration. Differentially Flat systems are useful in situations where explicit trajectory generation is required. Since the behavior of Flat system is determined by the Flat outputs (as previously defined), we can plan trajectories in output space, and then map them to appropriate inputs. On the other hand, while this technique is quite powerful, the application differential algebraic results to systems with strong geometric structure while at the same time exploiting that structure remains a difficult issue without simplifications.

In the following, some theoretical recalls on the differential flatness control and estimation to better understand the collaborative work achieved with our colleagues from "Mines-Paritech".

\subsection{Basic definitions}

Definition 1 (Flat system)

For continuous-time systems, differential flatness is defined as follows. Let us Consider the system:

$$
\dot{x}=f(x, u)
$$

where $x=\left(x, \cdots, x_{n}\right) \in \mathbb{R}^{n}$ and $u=\left(u, \cdots, u_{m}\right) \in \mathbb{R}^{m}$. It is said to be differentially flat (see [8], [9], [10] and [22], [23]) if, and only if:

- there exists a vector-valued function $h$ such that

where $y=\left(y, \cdots, y_{m}\right) \in \mathbb{R}^{m}, r \in \mathbb{N}$;

$$
y=h\left(x, u, \dot{u}, \cdots, u^{(r)}\right)
$$

- the components of $x=\left(x, \cdots, x_{n}\right)$ and $u=\left(u, \cdots, u_{m}\right)$ may be expressed as

$$
\begin{aligned}
& x=A\left(y, \dot{y}, \cdots, y^{\left(r_{x}\right)}\right), \quad r_{x} \in \mathbb{N} \\
& u=B\left(y, \dot{y}, \cdots, y^{\left(r_{u}\right)}\right), \quad r_{u} \in \mathbb{N}
\end{aligned}
$$

Remember that $y$ in Eq. (37) is called a flat output.

\section{Remark 5}

It is worth noticing that given a flat system, the number of components of a flat output is equal to the number of independent inputs.

Also, let us recall some properties of the flat systems, that may be useful in the following work (proofs can be found easily in [22]):

- A flat system is locally reachable.

- A linear system is flat if and only if it is controllable.

- Every flat system is endogenous dynamic feedback linearizable. Conversely, every endogenous dynamic feedback linearizable system is Flat.

In this study, the flatness property is used for both control and estimation purposes. Indeed, in the following thesis works, the algebraic estimation is used in this thesis study for the road profile estimation. 


\subsection{A short summary on the algebraic observer}

The estimation method uses the algebraic framework devoted for the design of algebraic observers with unknown inputs [30], [31], [32], [33]. The estimation approach uses also the algebraic identification methods for the numerical differentiation of noisy signals [32], [12]. The estimation with unknown input is based on the following properties:

\section{Definition 2}

[30], [31]

Consider the following nonlinear model:

$$
\begin{aligned}
& \dot{x}(t)=f(x(t), u(t)) \\
& y(t)=h(x(t))
\end{aligned}
$$

where $x(t)$ is the state vector, $u(t)$ is the input, $y(t)$ is a smooth output and $f$ is continuously differentiable and $f(0 ; 0)=0$. For the following, we assume that the input $u(t)$ and the output $y(t)$ are continuously differentiable for all $t \geq 0$.

The model (33) is said to be algebraically observable if there exist the integers $n_{1}>0$ and $n_{2}>0$ such that

$$
x(t)=\Gamma\left(y, \dot{y}, \cdots, y^{\left(n_{1}\right)}, u, \dot{u}, \cdots, u^{\left(n_{2}\right)}\right)(t)
$$

where $\Gamma(t)$ is a differentiable real-valued function of the outputs $y(t)$, the inputs $u(t)$, and their derivatives.

\section{Definition 3}

\section{[32], [33]}

An unknown input (or a fault) $f_{a}$ is said to be diagnosable if it is possible to estimate the unknown input $f_{a}$ from the measured outputs of the system. It means in other words that the unknown input $f_{a}$ is diagnosable if it is algebraically observable, i.e, in general

$$
P(f, u, \dot{u}, \cdots, y, \dot{y}, \cdots)=0
$$

then, the reconstructor (35) can may be used to estimate the unknown input (or a fault) $f$.

\section{Proposition 1}

the algebraic observability of any nonlinear system with unknown inputs is equivalent to express the dynamical state and the unknown inputs as functions of the inputs, the measured outputs and their finite time derivatives.

\section{Proposition 2}

A system is said observable with unknown inputs if, any state variable or an input variable, can be formulated as a function of the output and their finite time derivatives. This function can be called as an input-free estimator. It means in other words that an input-output system is observable with unknown input if, and only if, its zero dynamics is trivial. In addition, if the system is square, then the system is called flat $\|$ system with its flat output.

\section{Property 1}

Consider the system

$$
\dot{x}=f(x, u)
$$

where $x=\left(x, \cdots, x_{n}\right) \in \mathbb{R}^{n}$ and $u=\left(u, \cdots, u_{m}\right) \in \mathbb{R}^{m}$. It is said to be differentially flat (see [8], [9], [10] and [22], [23]) if, and only if:

\footnotetext{
"The differential flatness property of nonlinear systems in a differential algebraic context was introduced by [9], [10],[22], [23] 
- there exists a vector-valued function $h$ such that

$$
y=h\left(x, u, \dot{u}, \cdots, u^{(r)}\right)
$$

where $y=\left(y, \cdots, y_{m}\right) \in \mathbb{R}^{m}, r \in \mathbb{N}$;

- the components of $x=\left(x, \cdots, x_{n}\right)$ and $u=\left(u, \cdots, u_{m}\right)$ may be expressed as

$$
\begin{aligned}
& x=A\left(y, \dot{y}, \cdots, y^{\left(r_{x}\right)}\right), \quad r_{x} \in \mathbb{N} \\
& u=B\left(y, \dot{y}, \cdots, y^{\left(r_{u}\right)}\right), \quad r_{u} \in \mathbb{N}
\end{aligned}
$$

Remember that $y$ in Eq. (37) is called a flat output.

The above properties are equivalent to previously presented definitions .

\subsection{A short definition of algebraic denoising and numerical differentiation}

The numerical estimators ${ }^{*}$ (43) are deduced from operational calculation and algebraic manipulations. For this, consider the following real-valued polynomial time function $x_{N}(t) \in \mathbb{R}[t]$ of degree $N$

$$
x_{N}(t)=\sum_{\nu=0}^{N} x^{(\nu)}(0) \frac{t^{\nu}}{\nu !}, t \geq 0 .
$$

In the operational domain ${ }^{\dagger \dagger}$ (see e.g. [34]), (40) becomes

$$
X_{N}(s)=\sum_{\nu=0}^{N} \frac{x^{(\nu)}(0)}{s^{\nu+1}} .
$$

Multiplying the left-side and the right-side of equation (41) on the left by $\frac{d^{\alpha}}{d s^{\alpha}} s^{N+1}, \alpha=$ $0,1, \cdots, N$. The quantities $x^{(\nu)}(0), \nu=0,1, \ldots, N$, which are linearly identifiable satisfy the following triangular system of linear equations:

$$
\frac{d^{\alpha} s^{N+1} X_{N}}{d s^{\alpha}}=\frac{d^{\alpha}}{d s^{\alpha}}\left(\sum_{\nu=0}^{N} x^{(\nu)}(0) s^{N-\nu}\right), 0 \leq \alpha \leq N-1 .
$$

The time derivatives in (42) $\left(s^{\mu} \frac{d^{\iota} X_{N}}{d s^{\iota}}, \mu=1, \ldots, N, 0 \leq \iota \leq N\right)$, are removed by the multiplying both sides of equation (42) by $s^{-\bar{N}}, \bar{N}>N$. Now, consider an analytic time function, defined by the power series $x(t)=\sum_{\nu=0}^{\infty} x^{(\nu)}(0) \frac{t^{\nu}}{\nu !}$, which is assumed to be convergent around $t=0$. Approximate $x(t)$ by the truncated Taylor expansion $x_{N}(t)=\sum_{\nu=0}^{N} x^{(\nu)}(0) \frac{t^{\nu}}{\nu !}$ of order $N$. Good estimates of the derivatives are obtained by the same calculations as above.

Based on this development and after some simple mathematical manipulations, the following formulae may be obtained and used to estimate the $1^{\text {st }}$ order derivative of $y$ :

$$
\hat{\dot{y}}(t)=-\frac{3 !}{h^{3}} \int_{t-h}^{t}(2 h(t-\tau)-h) y(\tau) d \tau
$$

Note that the sliding time window $[t-h, t]$ may be quite short.

\section{Remark 6}

The estimation methods (43) is not of asymptotic type and do not require any statistical knowledge of the corrupting noises (see [13] for details).

\footnotetext{
** For the details related to the developments used in this work, we refer the reader to [32], [12]

$\dagger \dagger \frac{d}{d s}$ corresponds in time domain to the multiplication both sides by $-t$.
} 
1. B. Németh and P. Gáspár, "Design of actuator interventions in the trajectory tracking for road vehicles," in In Proceedings of the 51th IEEE Conference on Decision and Control (CDC), Orlando, Florida, USA, December, 2011.

2. H. Chou and B. d'Andréa Novel, "Global vehicle control using differential braking torques and active suspension forces," Vehicle Syst. Dynamics, vol. 43, pp. 261-284, 2005.

3. M. Doumiati, O. Sename, L. Dugard, J.-J. Martinez-Molina, P. Gaspar, and Z. Szabo, "Integrated vehicle dynamics control via coordination of active front steering and rear braking," European Journal of Control, vol. 19(2), pp. 121-143, March 2013.

4. B. L. Boada, M. Jesús, L. Boada, and V. Díaz, "Discussion on "integrated vehicle dynamics control via coordination of active front steering and rear braking"," European Journal of Control, vol. 19(2), pp. 144-145, March 2013.

5. P. Gáspár, Z. Szabó, J. Bokor, C. Poussot-Vassal, O. Sename, and L. Dugard, "Toward global chassis control by integrating the brake and suspension systems," in In Proceedings of the 5th IFAC Symposium on Advances in Automotive Control (AAC), Aptos, California, USA, August 2007.

6. S. Fergani, O. Sename, and L. Dugard, "A LPV/H ${ }_{\infty}$ global chassis controller for performances improvement involving braking, suspension and steering systems," in Proceedings of the 7th IFAC Symposium on Robust Control Design, Aalborg, Denmark, June 2012, pp. 363-368.

7. active suspension and steering systems," in Decision and Control (CDC), 2012 IEEE 51 st Annual Conference on, Dec 2012, pp. 4384-4389.

8. M. Fliess, J. Lévine, P. Martin, and P. Rouchon, "Sur les systèmes non linéaires différentiellement plats," Comptes rendus de l'Académie des sciences. Série 1, Mathématique, vol. 315, pp. 619-624, 1992.

9. - "Flatness and defect of nonlinear systems: introductory theory and examples," Int. J. Control, vol. 61, pp. $1327-1361,1995$

10. — "A lie-bäcklund approach to equivalence and flatness of nonlinear systems," IEEE Trans. Automat. Control, vol. 44, pp. 922-937, 1999.

11. M. Fliess, C. Join, and H. Sira-Ramírez, "Non-linear estimation is easy," Internat J. Modelling Identification Control, vol. 4, pp. 12-27, 2008.

12. M. Mboup, C. Join, and M. Fliess, "Numerical differentiation with annihilators in noisy environment," Numer. Algorithm., vol. 50, pp. 439-467, 2009.

13. M. Fliess, "Analyse non standard du bruit," C.R. Acad. Sci. Paris, vol. I-342, pp. 797-802, 2006.

14. T. Brown, A. Hac, and J. Martens, "Detection of vehicle rollover," in in SAE World Congress, Michigan-Detroit, December 2004.

15. W. F. Milliken and D. L. Milliken, Race car vehicle dynamics. Society of Automotive Engineers International, 1995.

16. L. Menhour and B. d' Andréa-Novel, "Survey and contributions in vehicle modeling and estimation," Rapport du projet INOVE, Tech. Rep., Oct. 2011.

17. L. Menhour, B. d'Andréa Novel, M. Fliess, and H. Mounier, "Coupled nonlinear vehicle control: Flatness-based setting with algebraic estimation techniques," Control Engineering Practice, vol. 22, pp. 135-146, 2014.

18. M. J. V. Nieuwstadt and R. M. Murray, "Real time trajectory generation for differentially flat systems," Int. J. Robust \& Nonlinear Control, vol. 8(11), pp. 995-1020, 1998.

19. L. Menhour, B. d'Andréa Novel, C. Boussard, M. Fliess, and H. Mounier, "Algebraic nonlinear estimation and flatness-based lateral/longitudinal control for automotive vehicles," in $14^{\text {th }}$ Int. IEEE Conf. ITS, Washington, 2011.

20. S. Fuchshumer, K. Schlacher, and T. Rittenschober, "Nonlinear vehicle dynamics control - a flatness based approach," in $44^{\text {th }}$ IEEE CDC \& ECC, Sevilla, 2005.

21. M. Rathinam, "Configuration flatness of lagrangian systems underactuated by one control," in Proceedings of the 35th IEEE Decision and Control, 11-13 Dec, 1996.

22. J. Lévine, Analysis and Control of Nonlinear Systems: A Flatness-based Approach. Springer, 2009.

23. H. Sira-Ramírez and S. Agrawal, Differentially Flat Systems. Marcel Dekker, 1993.

24. J. Villagra, B. d' Andréa-Novel, S. Choi, M. Fliess, and H. Mounier, "Robust stop-and-go control strategy: An algebraic approach for nonlinear estimation and control," International Journal of Vehicle Autonomous Systems, vol. 7, pp. 270-291, 2009.

25. J. Villagra, B. d' Andréa-Novel, M. Fliess, and H. Mounier, "A diagnosis-based approach for tire-road forces and maximum friction estimation," Control Engineering Practice, vol. 19, pp. 174-184, 2011.

26. F. G. Collardo, B. d'Andréa Novel, M. Fliess, and H. Mounier, "Analyse fréquentielle des dérivateurs algébriques," in XXIIe Colloque GRETSI, 2009.

27. A. L. Do, O. Sename, and L. Dugard, Control of Linear Parameter Varying Systems with Applications. Springer US, 2012, ch. LPV modeling and control of semi-active in automotive systems, pp. 381-411.

28. C. Poussot-Vassal, O. Sename, L. Dugard, P. Gáspár, Z. Szabó, and J. Bokor, "Attitude and handling improvements through gain-scheduled suspensions and brakes control," Control Engineering Practice, vol. 19, no. 3, pp. 252 $263,2011$.

29. S. Fergani, O. Sename, and L. Dugard, "An lpv/Ho ${ }_{\infty}$ integrated vehicle dynamic controller," IEEE Transactions on Vehicular Technology, vol. PP, no. 99, pp. 1-1, 2015.

30. J. P. Barbot, M. Fliess, and T. Floquet, "An algebraic framework for the design of nonlinear observers with unknown inputs," in 46 $6^{\text {th }}$ IEEE Conf. Decision Control, New Orleans, 2007.

31. J. Daafouz, M. Fliess, and G. Millérioux, "Une approche intrinsèque des observateurs linéaires à entrées inconnues," in Conf. int. francoph. automatique, Bordeaux, 2006.

32. M. Fliess and C. Join, "Commande sans modèle et commande à modèle restreint," $e$-STA, vol. 5, pp. 12-27, 2008.

33. S. Ibrir, "Online exact differentiation and notion of asymptotic algebraic observers," IEEE Transactions on Automatic Control, vol. 48(11), pp. 922-937, 2003. 
34. K. Yosida, Operational Calculus: A Theory of Hyperfunctions. Springer, New York, translated from Japanese, 1984. 